\title{
Uma visão diferenciada sobre o ensino de forças impulsivas usando um smartphone
}

A further look about teaching impulsive forces using a smartphone

\author{
V.L.B. de Jesus*1, D.G.G. Sasaki ${ }^{2}$ \\ ${ }^{1}$ Instituto Federal de Educação, Ciência e Tecnologia do Rio de Janeiro, Campus Nilópolis, Nilópolis, RJ, Brasil \\ ${ }^{2}$ Centro Federal Educação Tecnológica Celso Suckow da Fonseca, Unidade Maracanã, Rio de Janeiro, RJ, Brasil
}

Recebido em 4 de agosto de 2015. Aceito em 30 de setembro de 2015

\begin{abstract}
Os livros didáticos introdutórios sobre mecânica clássica são extremamente sucintos na abordagem de forças impulsivas e do conceito de impulso. Isso pode ser justificado pela ênfase no estudo de situações onde as forças aplicadas são todas constantes ou nas forças dependentes da posição, mas constantes no tempo, que são uma classe das chamadas de forças conservativas. Nas ocasiões que são abordadas as forças impulsivas, em geral exemplificadas por um golpe rápido em uma bola de golfe, de tênis ou de beisebol, o enfoque ainda é transformar a força variável no tempo em uma força média constante, cujo impulso é equivalente ao da força real. Neste trabalho propomos uma experiência simples de aplicar com os dedos uma força impulsiva sobre um smartphone e registrar a dependência temporal da força através do seu acelerômetro interno. Essa tecnologia possibilita a obtenção dos valores da velocidade durante a aplicação da força impulsiva por iteração dos dados extraídos de aceleração e tempo. Alguns aspectos interessantes negligenciados nos livros emergem dessa análise, tais como a ausência de simetria da força impulsiva aplicada, a possibilidade de modelar a interação Fcompleta, sem recorrer ao conceito de força média e o cálculo do impulso diretamente pelo teorema do impulso e do momento linear. Como consequência do experimento, ainda é possível obter o coeficiente de atrito cinético entre o smartphone e a superfície horizontal.
\end{abstract}

Palavras-chave: ensino de física, forças impulsivas, acelerômetro, smartphone.

The introductory textbooks on classical mechanics are extremely brief in addressing impulsive forces and the concept of impulse. This can be explained by the emphasis on the study of physical situations in which the applied forces are all constant or position dependent, but constant in time, which is the class of the so-called conservative forces. When the impulsive forces are referred to, they are generally illustrated by a quick blow on a ball, as in golf, tennis or baseball. The focus is still to turn the time dependent force into a constant average force, whose impulse is equivalent to the real force. This paper proposes a simple experiment that consists in applying with the fingers an impulsive force on a smartphone and record the time dependence of the force through its internal accelerometer. This technology allows obtaining the values of the speed during the applied impulsive force by iterating the data extracted of acceleration and time. Some interesting aspects neglected in books emerge from this analysis, such as the lack of symmetry of the applied impulsive force, the possibility of modeling the full interaction without appeal to the concept of average force and calculating impulse directly by impulse and linear momentum theorem. As a consequence of the experiment, it is also possible to obtain the kinetic friction coefficient between the smartphone and the horizontal surface.

Keywords: physics teaching, impulsive forces, accelerometer, smartphone.

\section{Introdução}

Em geral, os livros didáticos introdutórios de mecânica clássica são extremamente sucintos na

*Endereço de correspondência: vitor.jesus@ifrj.edu.br. abordagem de impulso e forças impulsivas [1-4]. A maioria deles sequer apresenta uma definição formal de força impulsiva, isto é aquela cuja intensidade é significativamente superior a qualquer outra força que atue sobre o corpo durante um curto intervalo 
de tempo, mas insignificante fora deste intervalo. A grandeza impulso costuma ser definida somente no contexto de colisões unidimensionais, muito embora alguns autores ressaltem que o impulso pode ser calculado para qualquer força, inclusive as forças constantes e conservativas

Em seguida, mostra-se que o impulso é numericamente equivalente à área sob a curva em um gráfico da força pelo tempo, que por sua vez corresponde, de forma simplificada, à área de um retângulo formado pela força média e o intervalo de tempo. Cabe destacar que somente uma das coleções [2] mostra um gráfico de força em função do tempo assimétrico, mas esse detalhe não é comentado no texto. Os livros prosseguem fazendo a demonstração do teorema do impulso e variação do momento linear. Os exercícios seguem a mesma linha: uma bola de golfe, tênis ou beisebol é golpeada, a massa, as velocidades finais e iniciais da bola são informadas e aplica-se o teorema do impulso para a obtenção da força média

Do ponto de vista didático, acreditamos que a abordagem tradicional é insatisfatória. Realmente, o conceito de força média é artificial e de pouca aplicação prática. A interação costuma ser tratada como uma caixa preta onde só temos acesso a sua duração e seus efeitos cinemáticos, isto é a variação total de velocidade. O impulso nunca é calculado diretamente pela sua definição, pois a força variável é essencialmente desconhecida. Logo, o teorema do impulso e momento linear se torna uma mera identidade formal. Alguns livros de física matemática sugerem que como a força impulsiva expressa as características de um golpe (blow), portanto não pode ser representada por uma função bem comportada [5]. A força impulsiva é reduzida ao delta de Dirac (valor infinito, mas de duração infinitesimal), o que acarreta uma substancial perda de informação sobre o processo de interação

Neste trabalho propomos e analisamos uma experiência, onde é viável modelar a força impulsiva numericamente através de medidas da aceleração instantânea. Assim, os valores das velocidades instantâneas durante a ação da força impulsiva podem ser calculados por iteração. Logo, o momento linear também pode ser conhecido durante e depois da interação. Tal fato acarreta em duas consequências: a primeira é tornar desnecessário o conceito de força média, visto que a força pode ser mapeada integralmente. A segunda é que o impulso pode ser calculado diretamente, em qualquer intervalo de tempo, usando o teorema do impulso e o momento linear, ou seja, através do produto da massa do corpo pelo correspondente numérico da área do gráfico de aceleração versus tempo

Para implementar essa proposta é necessário dispor de um sensor de aceleração que faça medidas em intervalos de tempo da ordem de milissegundos. Esses sensores podem ser adquiridos em empresas de equipamentos didáticos de laboratório. Contudo, nos últimos anos tem havido uma explosão de artigos sobre smartphone physics, isto é, uma linha de pesquisa em ensino que explora o uso dos sensores embutidos nos aparelhos de celulares que usam o sistema Android ou iOS da Apple. Em especial, citamos os trabalhos que envolvem o cálculo da aceleração da gravidade [6,7], o pêndulo simples [8], osciladores harmônicos e amortecidos $[9,10]$, rotações sobre um eixo fixo [11-14], momento angular [15] e impulso [16].

$\mathrm{O}$ aspecto original relevante deste trabalho é mostrar que é possível modelar numericamente algumas forças impulsivas que fazem parte do cotidiano e obter resultados das grandezas físicas com boa precisão e acurácia, usando como única ferramenta o sensor de aceleração interno de um smartphone

\section{Procedimento experimental}

Neste experimento foi utilizado o aplicativo gratuito Accelerometer Monitor, versão 1.6.0 que pode ser facilmente baixado pelo sítio da Google Play [17] que pode acessar os dados disponíveis no acelerômetro interno instalado em quase todos os smartphones à venda atualmente. As três componentes de aceleração podem ser obtidas no referencial do smartphone, que se torna automaticamente um referencial não inercial [18], sendo o eixo $y$ longitudinal ao aparelho, o eixo $x$ transversal e o eixo $z$ vertical, considerando o smartphone com a tela voltada para cima. As medidas são adquiridas em intervalos de tempo médio de $10 \mathrm{~ms}$ (fastest mode). A precisão é de $0,00059 \mathrm{~m} / \mathrm{s}^{2}$ e o limite superior de medição em módulo é de $20 \mathrm{~m} / \mathrm{s}^{2}$.

$\mathrm{O}$ funcionamento interno de um acelerômetro pode ser entendido, de uma maneira muito simplificada, através de uma analogia mecânica de uma caixa fechada contendo no seu centro uma esfera acoplada por três pares de molas paralelas aos eixos $x, y$ $e z$. Por exemplo, qual seria leitura do acelerômetro se a caixa fosse posicionada de forma que o eixo $\mathrm{z}$ 
estivesse na vertical? Nesse caso, teríamos a mola posicionada abaixo da esfera comprimida, enquanto a mola acima estaria distendida. Essa diferença na elongação das molas no eixo z pode ser interpretada como o acelerômetro (formado pela esfera, molas e caixa) acelerando para cima com módulo $g$, apesar de o sistema estar em repouso! A medida seria idêntica caso o sistema estivesse acelerando com módulo $g$ no plano horizontal na direção x. Em casos em que a medida de aceleração vertical é o objetivo, deve-se subtrair o valor de $g$ obtido enquanto o smartphone estiver em repouso sobre uma superfície plana e horizontal (em alguns aplicativos esta operação pode ser requisitada automaticamente). Neste trabalho não precisamos nos preocupar com isso, pois todas as medidas são realizadas no plano horizontal. Uma descrição mais avançada dos dispositivos internos de um acelerômetro, baseados em capacitores variáveis calibrados com massas de prova, pode ser encontrada na referência [19].

O aparato experimental era composto somente por uma mesa de madeira laqueada encerada e um smartphone Samsung Galaxy S4 modelo GT-I9515 de massa 130 gramas. A mesa pode ser substituída por uma placa de vidro ou qualquer superfície plana e polida. Para verificar o nivelamento da superfície foi baixado o aplicativo Bubble level versão 1.9.7 pelo sítio da Google Play. Existem aplicativos similares para aparelhos que usam o sistema operacional iOS.

$\mathrm{O}$ aparelho deve ser posicionado com o visor voltado para cima. Com o aplicativo Bubble level, verifica-se que a mesa está nivelada em um ângulo zero. A direção longitudinal do smartphone (da sua maior dimensão) coincide com a direção do eixo y e deve ser alinhada paralelamente à borda da mesa. Com o aparelho ainda em repouso, o alinhamento é ajustado com aplicativo Accelerometer Monitor, minimizando os valores da aceleração de fundo em todas as direções. O valor médio da aceleração de fundo (background) na direção do eixo y na experiência foi $a_{b g}=0,13 \mathrm{~m} / \mathrm{s}^{2}$.

$\mathrm{Na}$ execução do experimento existem dois pontos críticos que devem ser observados. O primeiro é minimizar a rotação do aparelho quando se aplica um golpe com os dedos. É fundamental que o movimento seja unidimensional para garantir que as medidas do acelerômetro não sejam contaminadas por um efeito centrífugo. O segundo cuidado é com a intensidade da força aplicada. A força não pode ser muita fraca, pois precisa superar a força de atrito estático e fazer o smartphone percorrer alguns centímetros antes de parar, sob a ação da força de atrito cinético. Por outro lado, a força não pode ser muito forte, pois a aceleração não pode superar o valor limite do acelerômetro $(a=2 \mathrm{~g})$. Além disso, uma pancada forte também costuma ser rápida, semelhante ao delta de Dirac, o que inviabilizaria a modelagem numérica.

O experimento é extremamente rápido e pode ser repetido várias vezes se houver dificuldade de ajustar a intensidade do empurrão e o alinhamento da trajetória. O movimento completo durou menos de um segundo e o smartphone percorreu uma distância de aproximadamente $33 \mathrm{~cm}$. A interação impulsiva teve duração de $140 \mathrm{~ms}$. O sensor de aceleração efetuou medidas em intervalos de tempo da ordem de $10 \mathrm{~ms}$, o que permitiu uma modelagem razoável da força impulsiva e bem detalhada da força de atrito cinético.

\section{Análise dos resultados}

Os dados experimentais gerados pelo acelerômetro foram transferidos para um notebook e tabulados em um programa padrão de planilha eletrônica (Excel, do pacote Office da Microsoft). A Fig. 1 mostra o gráfico da componente $y$ da aceleração versus tempo para o movimento do smartphone, já subtraído o valor da aceleração de fundo, $a_{b g}$, que se refere ao valor medido antes de se iniciar o experimento. Um aspecto qualitativo que se destaca é a ausência de simetria da força impulsiva. Em geral, os livros didáticos ilustram forças impulsivas simétricas, típicas de colisões entre corpos rígidos ou de situações mediadas por forças elásticas. Contudo, intuitivamente forças aplicadas diretamente pelas mãos devem ser assimétricas.

A assimetria da força impulsiva também pode ser aferida pela quantidade de pontos experimentais. São apenas 5 medidas do lado esquerdo do ponto máximo da aceleração, mas são 9 pontos experimentais após o pico. Isso indica que a força varia mais rapidamente antes de atingir o ápice e depois decai em uma taxa mais lenta.

A força impulsiva teve duração de apenas $140 \mathrm{~ms}$ (intervalo de tempo entre os instantes 1,031 s e $1,171 \mathrm{~s})$, marcados entre os pontos que cruzam a linha horizontal que marca aceleração nula. Durante os próximos $730 \mathrm{~ms}$ (intervalo de tempo entre os instantes $1,171 \mathrm{~s}$ e $1,902 \mathrm{~s}$ ) o smartphone adquire uma aceleração negativa praticamente constante até 


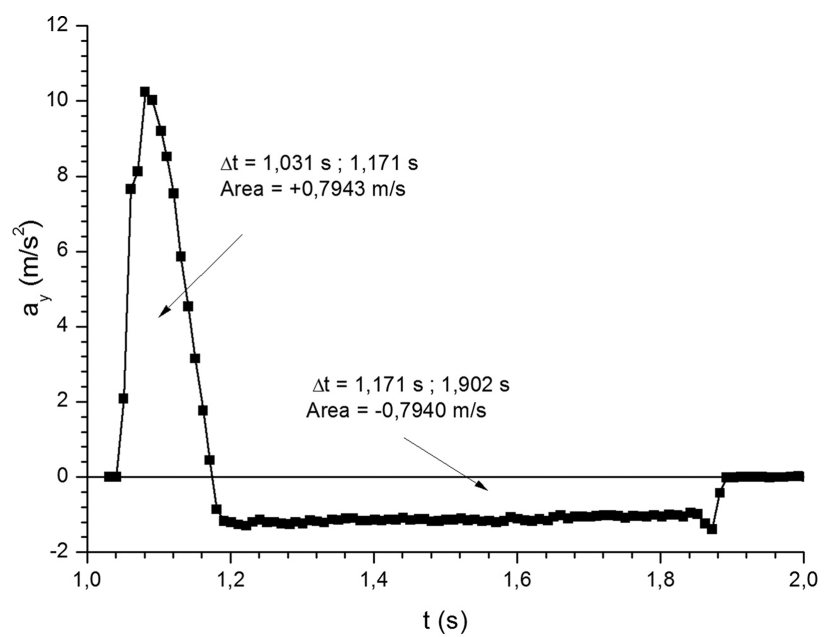

Figura 1: Gráfico da componente y da aceleração versus tempo. A força impulsiva tem duração de apenas $140 \mathrm{~ms}$ e a sua respectiva área sob o pico corresponde numericamente à variação de velocidade adquirida pelo smartphone. $\mathrm{Na}$ sequência, a aceleração fica constante em um valor médio negativo de $1,125(3) \mathrm{m} / \mathrm{s}^{2}$, indicando a ação da força de atrito cinético.

parar, indicando a ação da força de atrito cinético. Todo o movimento ocorre em um tempo inferior a 1 segundo.

A análise da Fig. 1 revela alguns aspectos bem curiosos. Como a massa do smartphone é constante, a relação entre diferentes valores da aceleração em cada instante fornece automaticamente a relação entre as forças resultantes que atuam no aparelho, em diferentes instantes. Então, pode-se concluir que a resultante das forças nos primeiros 140 ms contém um máximo que é $10,24 / 1,125 \approx 9$ vezes superior à força de atrito cinético. Além disso, a força impulsiva produz uma aceleração grande $(10,24+1,125=$ $11,365 \mathrm{~m} / \mathrm{s}^{2}$, superior à aceleração da gravidade. Sabendo que a massa do smartphone é de 130 gramas, então, através da segunda lei de Newton, constatamos que a força impulsiva tem um pico da ordem de $0,130 \mathrm{~kg} \times 11,365 \mathrm{~m} / \mathrm{s}^{2}=1,48 \mathrm{~N}$. Todas essas informações, bem como outras qualitativas sobre a assimetria da força, são perdidas na abordagem tradicional via força média.

Os valores das áreas equivalem numericamente à variação de velocidade do smartphone primeiramente devido à resultante entre a força impulsiva e a força de atrito cinético (área calculada sob a curva durante os $140 \mathrm{~ms}$ ) e depois exclusivamente pela última (área calculada sob a curva durante os próximos $730 \mathrm{~ms}$ ). As áreas foram calculadas utili- zando uma planilha eletrônica padrão e realizando o somatório

$$
\Delta v=\sum_{n}\left(a_{n}-a_{b g}\right) \Delta t_{n}
$$

onde cada termo do somatório é obtido da seguinte forma: a aceleração medida no instante $n, a_{n}$, subtraída do valor da aceleração de fundo, $a_{b g}$, é multiplicada pelo intervalo de tempo correspondente, $\Delta t_{n}$

Um bom indicativo da qualidade dos dados experimentais é o fato de que essas áreas têm valores numéricos opostos muito próximos $(+0,7943 \mathrm{~m} / \mathrm{s}$ e $-0,7940 \mathrm{~m} / \mathrm{s})$. De fato, a diferença percentual entre as áreas obtidas é de apenas 0,04\%, revelando que o acelerômetro produz medidas extremamente precisas e consistentes entre si.

Uma objeção pertinente é que o sensor na verdade mede a aceleração resultante proveniente da diferença entre a força impulsiva e a força de atrito cinético. Durante os $140 \mathrm{~ms}$ da interação impulsiva, também atua a força de atrito cinético, portanto os valores da aceleração da força impulsiva deveriam ser maiores daqueles efetivamente medidos. Ao contrário de ser algo inconveniente, essa constatação pode gerar um debate interessante em sala de aula. Os resultados após a ação da força impulsiva indicam que a força de atrito cinético provoca uma aceleração praticamente constante de - 1,125(3) m/s . Esse valor pode ser extrapolado para o movimento completo e serviria como uma correção aditiva da aceleração do smartphone, caso não houvesse força de atrito.

Os valores de velocidade foram obtidos isolando a velocidade na iteração numérica (equação 1), isto é, a velocidade do smartphone no instante $n$ é calculada a partir do produto da diferença entre a aceleração medida no instante $n, a_{n}$ e a aceleração de fundo $\left(a_{b g}\right)$ pelo intervalo de tempo entre $n$ e $n-1, \Delta t_{n}$ e adicionada ao valor da velocidade no instante anterior $n$-1. A velocidade no instante anterior ao início do impulso é considerada nula.

A Fig. 2 mostra o gráfico dos valores obtidos por iteração da componente $y$ da velocidade versus tempo, entre os instantes $1,0 \mathrm{~s}$ e $2,0 \mathrm{~s}$, que abrange o movimento completo.

Outra vantagem dessa modelagem em relação à abordagem tradicional da força média, é a possibilidade de calcular diretamente o impulso, em qualquer instante durante e após a interação impulsiva, 


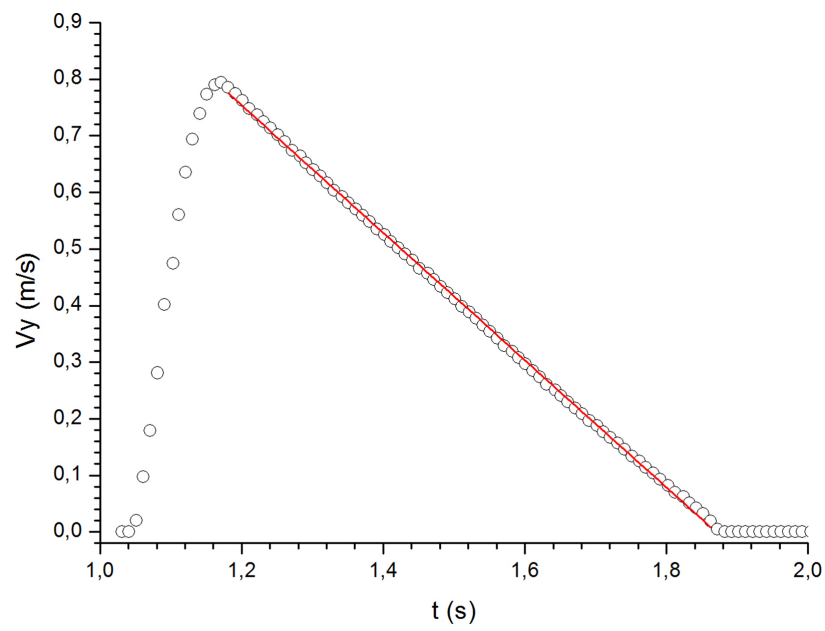

Figura 2: Gráfico da componente y da velocidade versus tempo entre os instantes $1,0 \mathrm{~s}$ e $2,0 \mathrm{~s}$. Os dados mostram que a velocidade declina em uma taxa constante de $1,125(3) \mathrm{m} / \mathrm{s}^{2}$, devido à ação da força de atrito cinético até atingir o repouso.

através do teorema do impulso e do momento linear. De fato, o teorema do impulso e momento linear é

$$
\mathbf{I}(t)=m \int_{0}^{t} \mathbf{a}\left(t^{\prime}\right) d t^{\prime}=m \Delta \mathbf{v}(t) .
$$

Além disso, pela Fig. 2, verifica-se que os valores da velocidade declinam em uma taxa constante de $1,125(3) \mathrm{m} / \mathrm{s}^{2}$, devido à ação da força de atrito cinético até chegar ao valor nulo. Os dados são precisos e permitem uma regressão linear com um ajuste $R$-quadrado de $99,94 \%$.

A análise da velocidade é reveladora sobre o que ocorre durante a interação impulsiva. A Fig. 3 mostra, em uma escala maior, o detalhe da Fig. 2, entre os instantes $1,0 \mathrm{~s}$ e $1,2 \mathrm{~s}$. O aumento da velocidade não é uniforme e pode ser dividido em três etapas. Na primeira, logo após o golpe, a velocidade varia numa taxa crescente, o que pode ser visualizado pelo aspecto curvo do gráfico com uma derivada mais acentuada. Em seguida, a velocidade aumenta em uma taxa constante, modelada por uma reta inclinada típica de um movimento uniformemente variado. Finalmente, a velocidade aumenta em uma taxa decrescente até atingir o valor máximo de $0,79 \mathrm{~m} / \mathrm{s}$, o que pode ser inferido da curva com uma derivada cada vez mais suave.

Um detalhe complementar, mas ainda assim interessante é a possibilidade de calcular o coeficiente de atrito cinético entre a parte debaixo do smartphone e a superfície de madeira laqueada encerada. O coe-

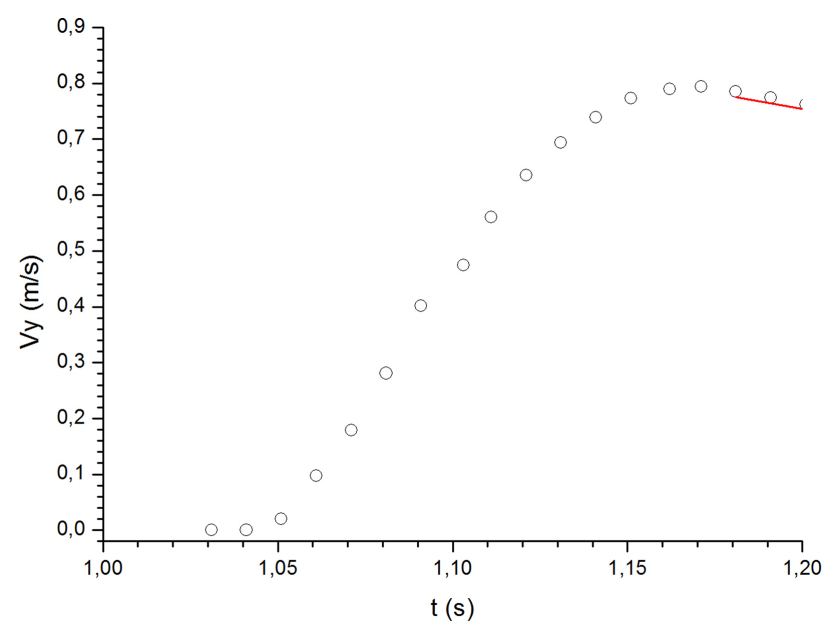

Figura 3: Gráfico da componente y da velocidade versus tempo entre os instantes $1,0 \mathrm{~s}$ e $1,2 \mathrm{~s}$. Os dados mostram que a velocidade aumenta numa taxa crescente logo após a aplicação da força impulsiva, depois sofre uma inflexão que pode ser modelada por uma reta inclinada e finalmente aumenta em uma taxa decrescente até atingir o valor máximo de $0,79 \mathrm{~m} / \mathrm{s}$.

ficiente de atrito cinético é obtido pela razão entre o módulo da inclinação da reta ajustada (desaceleração) e a aceleração da gravidade. No local, o valor padrão é $g=9,78777 \mathrm{~m} / \mathrm{s}^{2}$, o que fornece um coeficiente de atrito cinético $m=0,1149(3)$.

\section{Conclusões}

A modelagem numérica de forças impulsivas em determinados casos do cotidiano revelou-se viável e profícua do ponto de vista da compreensão das grandezas físicas envolvidas e de suas relações. Essa abordagem possibilitou uma análise da força impulsiva como um todo, isto é, o seu comportamento durante a interação, seu valor máximo, bem como os valores de aceleração e velocidade de uma partícula durante a interação. Isso representa um enorme ganho de informação e conteúdo se comparado com a abordagem tradicional onde a força impulsiva é considerada desconhecida ou modelada por uma função delta de Dirac.

Nessa abordagem, a noção artificial de força média foi abandonada e o teorema do impulso e do momento linear fica mais evidente. Realmente, numa interação cuja força é desconhecida, é impossível calcular o impulso pela sua definição. Além disso, o momento linear após a interação também não pode ser previsto teoricamente, sendo acessível somente por medição direta. Neste trabalho mostramos que 
é possível, em algumas situações, modelar a força impulsiva com o uso do acelerômetro e como consequência, obter a velocidade instantânea e o momento linear em qualquer tempo, durante e após a interação.

Sem dúvida o resultado mais importante foi mostrar que é possível modelar numericamente algumas forças impulsivas e obter dados de algumas grandezas cinemáticas, com boa precisão e acurácia, usando apenas o sensor de aceleração interno de um smartphone.

\section{Referências}

[1] D. Halliday, R. Resnick e J. Walker, Fundamentos de Física (LTC, Rio de Janeiro, 2012), v. 1, 9a ed.

[2] P.A. Tipler e G. Mosca, Física para Cientistas e Engenheiros (LTC, Rio de Janeiro, 2009), v. 1, $6^{\text {a }}$ ed.

[3] H.M. Nussenzveig, Curso de Física Básica (Edgard Blucher, São Paulo, 2002), v. 1, 4 ${ }^{\mathrm{a}}$ ed

[4] H.D. Young e R.A. Freedman, Física I (Pearson, São Paulo, 2008), $12^{\mathrm{a}}$ ed.

[5] E. Butkov, Mathematical Physics (Addison-Wesley, New York, 1968), $1^{\text {a }}$ ed.

[6] J. Kuhn and P. Vogt, European Journal of Physics Education 4, 16 (2013).

[7] P. Vogt and J. Kuhn, The Physics Teacher 50, 182 (2012).

[8] P. Vogt and J. Kuhn, The Physics Teacher 50, 439 (2012).

[9] J.C. Castro-Palacio, L. Velázquez-Abad, M.H. Giménez and J.A. Monsoriu, American Journal of Physics 81, 472 (2013).

[10] J. Kuhn and P. Vogt, The Physics Teacher 50, 504 (2012).

[11] M. Monteiro, C. Cabeza and A.C. Marti, The Physics Teacher 52, 180 (2014).

[12] M. Monteiro, C. Cabeza, A.C. Marti, P. Vogt and J. Kuhn, The Physics Teacher 52, 312 (2014).

[13] M. Monteiro, C. Cabeza and A.C. Marti, European Journal of Physics 35, 045013 (2014).

[14] P. Vogt and J. Kuhn, The Physics Teacher 51, 182 (2013).

[15] A. Shakur and T. Sinatra, The Physics Teacher 51 564 (2013).

[16] J.W. Streepey, The Physics Teacher 51, 54 (2013).

[17] https://play.google.com/store/apps.

[18] M. Monteiro, C. Cabeza e A.C. Marti, Revista Brasileira de Ensino de Física 3, 1303 (2015).

[19] C.L. Countryman, The Physics Teacher 52, 557 (2014). 